\title{
Limitation of the resting ankle-brachial index in symptomatic patients with peripheral arterial disease
}

\author{
Russell Steina , Ingrid Hriljaca, Jonathan L Halperina, Susan M Gustavsona, \\ Victoria Teodorescub and Jeffrey W Olina
}

\begin{abstract}
Peripheral arterial disease (PAD) has been demonstrated to be prevalent in the primary care setting. However, it has also been shown to be unrecognized and under-treated. Owing to the association with cardiovascular disease it has been recommended to screen high-risk patients for PAD in the primary care setting using the ankle-brachial index ( $A B I)$. $A B I$ has been demonstrated to be highly sensitive and specific in diagnosing PAD in patients with significant stenosis. However, the utility in patients with less severe stenosis and calcified vessels is in question. The aims of this study were to determine the diagnostic utility of measuring the $A B I$ at rest in patients referred to the vascular laboratory for evaluation of suspected PAD, and to assess the added value of pulse volume recordings and post-exercise studies in patients with a normal ABI. A computerized vascular diagnostic laboratory database was queried for symptomatic outpatients referred for measurement of segmental blood pressure, the $\mathrm{ABI}$ or pulse volume recordings by physicians not specialized in the evaluation and management of patients with peripheral vascular disease. Of 707 patients undergoing outpatient physiologic arterial evaluations between February 1, 2003 and July 31, 2004, 396 met these inclusion criteria. Data recorded included resting $\mathrm{ABI}, \mathrm{ABI}$ following treadmill exercise test and the presence of abnormal pulse volume recordings. The study population $(n=396)$ consisted of equal numbers of men and women (mean age 69 years, range 19-100 years). Among 396 studies, resting $A B I$ values were normal in 183 (46.2\%) and abnormal in 159 (40.2\%). Of the 138 patients who underwent exercise testing, 84 had normal $A B I$ readings at rest. In the 84 patients who had a normal $A B I$ at rest and underwent exercise testing, the $\mathrm{ABI}$ fell below 0.9 after exercise in 26 (31\%). Arterial non-compressibility was detected in 54 (13.6\%) patients, whose average age was 67 years. Thirteen (24\%) of those with non-compressible vessels had abnormal pulse volume recording (PVR) results, compared to five with normal resting $A B I$ who had abnormal PVR findings (2.7\%). In conclusion, this study demonstrated that nearly half of patients referred to the outpatient vascular laboratory because of suspected arterial disease had a normal resting $A B I$. While it is recommended that the $A B I$ be measured at rest in patients at risk of PAD in primary care practice, these findings suggest that patients with symptoms of PAD should be more completely evaluated in a vascular laboratory. Furthermore, when the $A B I$ is normal at rest in patients with symptoms of intermittent claudication, exercise testing is recommended to enhance the sensitivity for detection of PAD.
\end{abstract}

Key words: claudication; peripheral arterial disease; vascular laboratory

aZena and Michael A Wiener Cardiovascular Institute and Marie-Josée and Henry R Kravis Center for Cardiovascular Health, Mount Sinai Medical Center, New York, NY USA; ${ }^{b}$ Department of Surgery, Division of Vascular Surgery, Mount Sinai School of Medicine, New York, NY, USA

Address for correspondence: Jeffrey W Olin, Professor of Medicine, Director, Vascular Medicine, Mount Sinai School of Medicine, One Gustave L Levy Place, Box 1033, New York, NY 10029, USA. Tel +1 212241 9454; Fax: +1 212241 5107; E-mail: jeffrey.olin@msnyuhealth.org

Dr Thom W Rooke served as Guest Editor for this article.

\section{Introduction}

Peripheral arterial disease (PAD) affects 5 to 10 million adults in the USA. ${ }^{1,2}$ Patients with PAD have a 6.6-fold greater risk of death from coronary disease compared to patients without PAD and a 3.1-fold greater mortality from any cause. ${ }^{3}$ In the PARTNERS study of 6979 primary care patients, $29 \%$ of those who were either older than 70 years or between the ages of 50 and 69 years with a history of tobacco smoking or diabetes mellitus had PAD. ${ }^{4}$ Despite its known association with coronary artery disease, PAD was typically 
unrecognized or under-treated because many patients with PAD are asymptomatic or have atypical symptoms, and findings on physical examination may be subtle or misleading. 5,6

The lower the ankle-brachial index (ABI), the higher the cardiovascular mortality. ${ }^{4,5,7}$ It is recommended that the ABI be measured in high-risk patients to better identify patients who may benefit from more stringent cardiovascular risk factor modifications. ${ }^{5}$ When taken at rest, the ABI is highly specific (99\%) and sensitive (94-97\%) for the detection of high-grade stenosis as assessed by angiography. ${ }^{8-11}$ The ABI may underestimate the severity of PAD in patients with non-compressible vessels due to arterial calcification; ${ }^{12}$ however, most commonly in the presence of diabetes mellitus, end-stage renal disease or advanced age. ${ }^{13}$ In patients with arterial calcification, pulse volume recording (PVR) in the vascular laboratory overcomes this limitation of ABI measurement, with greater sensitivity for the diagnosis of PAD. ${ }^{9,14,15}$ Additionally, falsely negative results may arise in some patients with PAD who have normal ABI at rest but a fall in the ABI after limb exercise. ${ }^{16,17}$

Spurred by the results of the PARTNERS study and other initiatives, it is recommended that practitioners measure the $\mathrm{ABI}$ in the office in patients at risk of $\mathrm{PAD}$, raising questions about the proper role of the vascular laboratory in diagnosis. The aims of this study were to determine the diagnostic utility of measuring the $\mathrm{ABI}$ at rest in patients referred to the vascular laboratory for evaluation of suspected PAD, and to assess the added value of PVR and post-exercise studies in patients with a normal ABI. The results may help guide more appropriate outpatient use of vascular diagnostic laboratory testing by general physicians.

\section{Methods}

In this retrospective and observational study, the vascular diagnostic laboratory database was queried for symptomatic outpatients referred for measurement of segmental blood pressures, ABI and PVRs by physicians not specialized in the evaluation and management of patients with peripheral vascular disease. All vascular studies were performed at the Marie-Josée and Henry R Kravis Vascular Diagnostic Laboratory in the Zena and Michael A Wiener Cardiovascular Institute of the Mount Sinai School of Medicine. The laboratory is accredited by the Intersocietal Commission for the Accreditation of Vascular Laboratories and all of the technologists and medical directors (VT, JWO) are Registered Vascular Technologists. Of 707 patients undergoing outpatient physiologic arterial evaluations between February 1, 2003 and July 31, 2004, 396 met these inclusion criteria. Vascular specialists were defined as physicians who predominantly practiced vascular surgery, vascular medicine, cardiovascular medicine with an emphasis on peripheral vascular diseases or interventional radiology. Indications for testing included intermittent claudication, cutaneous ulceration, rest pain, decreased pulses or cold feet.

The ABI was measured in both legs by sphygmomanometry, using an $8 \mathrm{MHz}$ continuous-wave Doppler ultrasound transducer. The higher of the posterior tibial (PT) or dorsalis pedis (DP) artery systolic blood pressure values was taken as the numerator for each leg and the higher of the right and left brachial systolic blood pressure readings as the denominator. An ABI $\leq 0.90$ in either leg was considered abnormal. Arterial non-compressibility was diagnosed when the Doppler systolic signal was not obliterated at ankle cuff inflation pressures $\geq 250 \mathrm{mmHg}$.

Exercise evaluations were performed at the request of referring physicians. Ankle and brachial arterial pressure measurements were made at rest and repeated immediately after walking for 5 minutes on a treadmill at $12 \%$ grade and $2.0 \mathrm{mph}(3.2 \mathrm{k} / \mathrm{h})$ or until symptoms forced the patient to stop. A post-exercise ABI $\leq 0.90$ was considered abnormal. Segmental PVR was measured in the lower limbs using a standard protocol. ${ }^{18}$ Any prolongation or attenuation of the pulse volume waveforms were considered abnormal.

\section{Results}

The study population $(n=396)$ consisted of equal numbers of men and women (mean age 69 years, range 19-100 years). Among 396 studies, resting ABI values were normal in $183(46.2 \%)$, abnormal in 159 $(40.2 \%)$ and non-compressible in 54 (13.6\%). The resting $\mathrm{ABI}$ values based on indications for testing in each referral group are shown in Figure 1.

Some patients with normal ABI did not undergo exercise testing because of their inability to exercise, severe cardiac disease, or physician or patient refusal. Of the 138 patients who underwent exercise testing, 84 had normal ABI readings at rest. Most of those with normal resting $\mathrm{ABI}$ values who underwent exercise tests $(71.4 \%)$ had been referred because of intermittent claudication (Figure 2). In the 84 patients who had a normal $\mathrm{ABI}$ at rest and underwent exercise testing, the ABI fell below 0.9 after exercise in $26(31 \%)$.

Arterial non-compressibility was detected in 54 $(13.6 \%)$ patients, whose average age was 67 years. Thirteen (24\%) of those with non-compressible vessels had abnormal PVR results, compared to five with normal resting ABI who had abnormal PVR findings (2.7\%).

\section{Discussion}

Although PAD is prevalent, there are no exerciseinduced leg symptoms in approximately half of the 


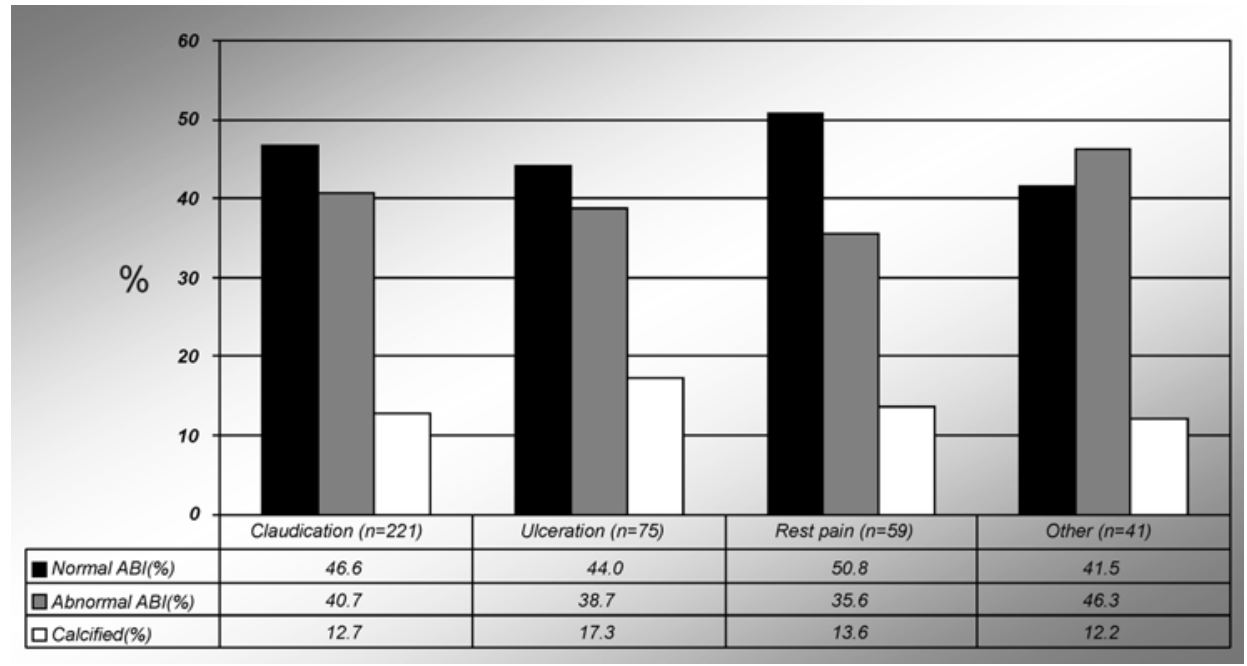

Figure 1 Resting ankle-brachial index values in relation to the indication for study.

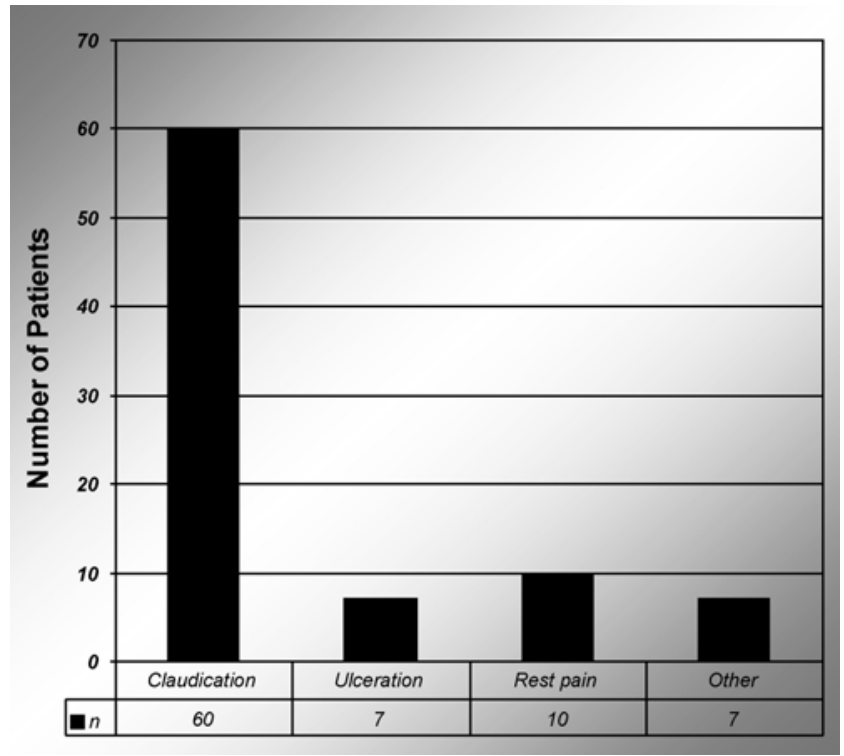

Figure 2 Indications for exercise testing in patients with a normal resting ankle-brachial index.

cases and the diagnosis is often overlooked in clinical practice. ${ }^{6}$ The Rose criteria ${ }^{19}$ for intermittent claudication (exertional pain involving the calf that impedes walking, resolves within 10 minutes of rest, and neither begins at rest nor resolves on walking) are satisfied in fewer than $10 \%$ of patients with $\mathrm{PAD}^{5}$ and the femoral pulse is preserved in about $87 \% .^{6}$ The difficulty in using clinical symptoms to diagnose PAD is demonstrated by the fact that, among the study population, $47 \%$ of those with symptoms suspicious for claudication (51\% of patients, with rest pain and $44 \%$ referred for ulceraction) had normal ABI values. The patients referred for claudication with normal resting ABI likely had either lesser degrees of stenosis that were not detected with resting $\mathrm{ABI}$ alone or symptoms simulating claudication but due to some other cause.
However, the normal resting ABI values in patients with rest pain or ulceration in all likelihood reflect errors in diagnosis by the referring non-vascular specialist.

An ABI value $<0.9$ correlates with angiographic evidence of obstructive PAD; sensitivity approaches 95\% and specificity is almost perfect for the exclusion of patients without the disease. ${ }^{8-11,20}$ Few contemporary studies have evaluated the accuracy of the ABI for diagnosis of PAD in the broad spectrum of patients encountered in general clinical practice. Older studies were limited to cases with relatively advanced PAD. In a study of 146 limbs with angiographically documented atherosclerosis, for example, 112 (77\%) had complete arterial occlusion; systolic blood pressure at the ankle was below normal in all cases. ${ }^{11}$ In the 85 control limbs without angiographically apparent stenosis, the ABI was $\geq 0.99$. The ABI was less accurate across the middle part of the spectrum in 34 limbs with mild to severe arterial stenosis; abnormal in $44 \%$ of limbs with mild stenosis and $76 \%$ of those with severe stenosis. ${ }^{11}$ The predictive value of reduced ABI measurements for the detection of PAD of lesser severity is limited, with no significant difference in $\mathrm{ABI}$ between asymptomatic patients with angiographically normal-appearing arteries and those with nonobstructive PAD. ${ }^{9}$ Exercise testing may enhance the detection of PAD in patients with non-obstructive PAD who have (falsely) normal ABI readings. ${ }^{10}$

The inability of resting ABI measurement to detect mild arterial stenosis results from preservation of perfusion pressure as described by Poisseuille's law governing flow across a zone of luminal narrowing in proportion to the 4 th power of the radius. ${ }^{21,22}$ During exercise, flow cannot increase beyond the zone of significant stenosis despite the reduction of resistance resulting from arteriolar dilatation, so blood pressure drops distally (Ohm's law). ${ }^{23} \mathrm{~A}$ fall in systolic 
pressure after exercise is the most sensitive test for detection of hemodynamically significant stenosis; thus, measurement of the ABI after exercise is most useful for the detection of PAD when stenosis is not severe enough to compromise perfusion at rest.

Of the patients who had normal ABI measurements at rest, $31 \%$ had abnormal readings after exercise. While angiographic documentation of the presence (and severity) or absence of PAD was not obtained, previous studies in patients with clinically suspected PAD and normal resting ABI readings have yielded conflicting results with respect to the utility of stress testing. In a study of 218 patients with PAD, stress testing increased the diagnostic yield by only $1.6 \%$ compared to $\mathrm{ABI}$ measurements at rest, and stress testing increased the yield by only $6 \%$ when rest and post-exercise ABI measurements were compared in the contralateral, asymptomatic limbs. The incremental yield rose to $22 \%$ when patients with intermittent claudication and normal resting ABI values were subjected to exercise testing. ${ }^{17}$ Taken together with our findings, available data support the value of exercise assessment in patients with exertional limb symptoms who have normal ABI at rest. Many patients with normal ABI did not undergo exercise testing. The reasons for this included an inability to exercise on a treadmill, pre-existing cardiac disease, or at the request of the referring physician.

An alternative to treadmill exercise testing is active pedal plantar flexion (heel raises). Heel raising exercises are carried out to a maximum height achievable by a patient at a maximum speed possible for $30 \mathrm{sec}-$ onds. Heel raising exercises produce changes in ankle pressure that correlate well with treadmill exercise. ${ }^{24}$

A relatively high proportion of patients $(13.6 \%)$ had arterial non-compressibility that produced falsely elevated ABI readings. In a previous study of over 600 limbs, Carter found the incidence of non-compressibility to be only $1 \% .{ }^{11}$ The Strong Heart Study demonstrated the value of identifying subjects with either abnormally high or low ABI measurements; both cardiovascular and all-cause mortality were lowest in patients with $\mathrm{ABI}$ values between 1.0 and 1.39. Risk-adjusted odds ratios for all-cause mortality were 1.69 (1.34-2.14) and 1.77 (1.48-2.13) for those with an ABI $<0.90$ and $>1.40$, respectively. Similar risk ratios for cardiovascular mortality were 2.52 for those in the low and 2.09 for the high ABI categories. $^{25}$

\section{Limitations}

This study of outpatients referred to the vascular laboratory has inherent limitations linked to its retrospective design. Eligibility was limited to patients referred by physicians not specially trained in the care of patients with vascular disease, and the potential for referral bias makes it difficult to generalize the findings to other populations. Only limited information was available about other clinical features such as diabetes or manifestations of atherosclerotic disease in vascular beds other than the lower extremities. Perhaps most importantly is the lack of angiographic confirmation of the presence or severity of PAD by which to assess the overall accuracy of the noninvasive laboratory evaluation.

\section{Conclusions}

This study demonstrated that nearly half of the patients referred by non-vascular specialists to the outpatient vascular laboratory because of suspected arterial disease had a normal ABI. Even patients referred because of rest pain and/or ulceration had a normal ABI $51 \%$ and $44 \%$ of the time, respectively. Of the 84 patients with normal ABI readings, exercise testing disclosed an abnormality consistent with PAD in almost a third. While it is recommended that the ABI be measured at rest in patients at risk of PAD in primary care practice, ${ }^{4}$ these findings suggest that patients with symptoms of PAD should be more completely evaluated in a vascular laboratory. Furthermore, when the ABI is normal at rest in patients with symptoms of claudication, exercise testing is recommended to enhance the sensitivity for the detection of PAD.

This study also demonstrated arterial noncompressibility in $13.6 \%$ of patients referred for ABI. PVRs were abnormal in $24 \%$ of this patient subgroup and thus it is a valuable diagnostic test, especially in patients with calcified vessels.

\section{References}

1 Selvin E, Erlinger TP. Prevalence of and risk factors for peripheral arterial disease in the United States: results from the National Health and Nutrition Examination Survey, 1999-2000. Circulation 2004; 110: 738-43.

2 Criqui MH. Peripheral arterial disease - epidemiological aspects. Vasc Med 2001; 6 (3 suppl): 3-7.

3 Criqui $\mathrm{MH}$, Langer RD, Fronek A et al. Mortality over a period of 10 years in patients with peripheral arterial disease. $N$ Engl J Med 1992; 326: 381-86.

4 Hirsch AT, Criqui MH, Treat-Jacobson D et al. Peripheral arterial disease detection, awareness, and treatment in primary care. JAMA 2001; 286: 1317-24.

5 McDermott MM, Kerwin DR, Liu K et al. Prevalence and significance of unrecognized lower extremity peripheral arterial disease in general medicine practice. J Gen Intern Med 2001; 16: 384-90.

6 Criqui MH, Fronek A, Klauber MR, Barrett-Connor E, Gabriel S. The sensitivity, specificity, and predictive value of traditional clinical evaluation of peripheral arterial disease: results from noninvasive testing in a defined population. Circulation 1985; 71: 516-22.

7 Papamichael CM, Lekakis JP, Stamatelopoulos KS et al. Ankle-brachial index as a predictor of the extent of coronary 
atherosclerosis and cardiovascular events in patients with coronary artery disease. Am J Cardiol 2000; 86: 615-18.

8 Carter SA. Clinical measurement of systolic pressures in limbs with arterial occlusive disease. JAMA 1969; 207: 1869-74.

9 Ouriel K, Zarins CK. Doppler ankle pressure: an evaluation of three methods of expression. Arch Surg 1982; 117: 1297-300.

10 Yao ST, Hobbs JT, Irvine WT. Ankle systolic pressure measurements in arterial disease affecting the lower extremities. Br J Surg 1969; 56: 676-79.

11 Carter SA. Indirect systolic pressures and pulse waves in arterial occlusive diseases of the lower extremities. Circulation 1968; 37: 624-37.

12 Quigley FG, Faris IB, Duncan HJ. A comparison of Doppler ankle pressures and skin perfusion pressure in subjects with and without diabetes. Clin Physiol 1991; 11: 21-25.

13 Taguchi JT, Suwangool P. "Pipe-stem" brachial arteries: a cause of pseudohypertension. JAMA 1974; 228: 733.

14 Kempczinski RF. Segmental volume plethysmography in the diagnosis of lower extremity arterial disease. J Cardiovasc Surg 1982; 23: 125-29.

15 Darling RC, Raines JK, Brener BJ, Austen WG. Quantitative segmental pulse volume recorder: a clinical tool. Surgery 1972; 6: 873-87.

16 Carter SA. Response of ankle systolic pressure to leg exercise in mild or questionable arterial disease. N Engl J Med 1972; 287: $578-82$.
17 Ouriel K, McDonnell AE, Metz CE, Zarins CK. Critical evaluation of stress testing in the diagnosis of peripheral vascular disease. Surgery 1982; 91: 686-93.

18 Sumner DS. Volume plethysmography in vascular disease: an overview. In Bernstein E ed. Vascular Diagnosis. Mosby, 1993: 181-93.

19 Rose GA. The diagnosis of ischaemic heart pain and intermittent claudication in field surveys. Bull World Health Organ 1962; 27: 645-58.

20 Belch JJ, Topol EJ, Agnelli G et al. Prevention of Atherothrombotic Disease Network. Critical issues in peripheral arterial disease detection and management: a call to action. Arch Intern Med 2003; 163: 884-92.

21 May AG, van De Berg L, Deweese JA, Rob CG. Critical arterial stenosis. Surgery 1963; 54: 250-59.

22 May AG, Deweese JA, Rob CG. Hemodynamic effects of arterial stenosis. Surgery 1963; 53: 513-24.

23 Cappelen C Jr, Hall KV. The effect of obstructive arterial disease on the peripheral arterial blood pressure. Registrations from the dorsalis pedis artery of normal persons and patients before and after operation. Surgery 1960; 48: 888-93.

24 Amirhamzeh MMR, Chant HJ, Rees JL, Hands LJ, Powell RJ, Campbell WB. A comparative study of treadmill tests and heel raising exercise for peripheral arterial disease. Eur J Vasc Endovasc Surg 1997; 13: 301-05.

25 Resnick HE, Lindsay RS, McDermott MM et al. Relationship of high and low ankle brachial index to all-cause and cardiovascular disease mortality: the Strong Heart Study. Circulation 2004; 109: 733-39. 